\title{
Compact Polarimetry Synthetic Aperture Radar Ocean Wind Retrieval: Model Development and Validation
}

\author{
Biao Zhang, ${ }^{\text {a,b,c }}$ Yiru Lu, ${ }^{\mathrm{a}}$ William Perrie, ${ }^{\mathrm{c}}$ Guosheng Zhang, ${ }^{\mathrm{a}, \mathrm{b}}$ ANd Alexis Mouche ${ }^{\mathrm{d}}$ \\ ${ }^{\text {a }}$ School of Marine Sciences, Nanjing University of Information Science and Technology, Nanjing, China \\ ${ }^{\mathrm{b}}$ Southern Marine Science and Engineering Guangdong Laboratory (Zhuhai), Zhuhai, China \\ ${ }^{\mathrm{c}}$ Bedford Institute of Oceanography, Fisheries and Oceans Canada, Dartmouth, Nova Scotia, Canada \\ ${ }^{\mathrm{d}}$ IFREMER, Université Brest, CNRS, IRD, Laboratoire d'Océanographie Physique et Spatiale, Brest, France
}

(Manuscript received 12 March 2020, in final form 23 November 2020)

\begin{abstract}
We have developed C-band compact polarimetry geophysical model functions for RADARSAT Constellation Mission ocean surface wind speed retrieval. A total of 1594 RADARSAT-2 images acquired in quadpolarization SAR imaging mode were collocated with in situ buoy observations. This dataset is first used to simulate compact polarimetric data and to examine their dependencies on radar incidence angle and wind vectors. We find that right circular transmit, right circular receive (RR-pol) radar backscatters are less sensitive to incidence angles and wind directions but are more dependent on wind speeds, compared to right circular transmit, horizontal receive (RH-pol), right circular transmit, vertical receive (RV-pol), and right circular transmit, left circular receive (RL-pol). Subsequently, the matchup data pairs are used to derive the coefficients of the transfer functions for the proposed compact polarimetric geophysical model (CMOD) functions, and to validate the associated wind speed retrieval accuracy. Statistical comparisons show that the retrieved wind speeds from CMODRH, CMODRV, CMODRL, and CMODRR are in good agreement with buoy measurements, with root-mean-square errors of $1.38,1.51,1.47$, and $1.25 \mathrm{~m} \mathrm{~s}^{-1}$, respectively. The results suggest that compact polarimetry is a good alternative to linear polarization for wind speed retrieval. CMODRR is more appropriate to retrieve high wind speeds than CMODRH, CMODRV or CMODRL.
\end{abstract}

KEYWORDS: Ocean; Wind; Algorithms; Remote sensing

\section{Introduction}

Satellite remotely sensed oceanic winds are of great value for marine forecasting and ocean modeling (Bourassa et al. 2019), investigating coupled ocean-atmosphere interactions (Chelton and Xie 2010), and examining global wind speeds and wave height trends (Young and Ribal 2019). Compared to satellite scatterometers and radiometers, spaceborne synthetic aperture radars (SARs) have the capability to map subkilometerscale ocean surface wind fields due to their high spatial resolution and wide coverage. Recently, the launch of the Canadian RADARSAT Constellation Mission (RCM) satellites on 12 June 2019 significantly improves the temporal resolution of SAR, and thus provides the possibility to reveal the variation of ocean winds over relatively short time intervals. Moreover, RCM has a new compact polarimetry (CP) configuration for various applications related to ocean observations, with abundant polarimetric content and large spatial coverage. These characteristics open up a new era of oceanic wind measurements from space.

RCM consists of three identical C-band SAR satellites on the same orbital plane. RCM is more advanced compared to previous RADARSAT satellites, because it can provide daily coverage to $95 \%$ of the global oceans, short revisit period, and $\mathrm{CP}$ imaging mode, which transmits right circular polarization and receives two orthogonal mutually coherent linear polarizations (Thompson 2015). CP SAR is a coherent dualpolarization (dual-pol) radar system retaining relative phase

Corresponding author: Biao Zhang, zhangbiao@nuist.edu.cn between the two receive polarizations, which has the capability of obtaining more abundant polarization information than the conventional dual-pol mode (Charbonneau et al. 2010). Moreover, CP SAR is relatively simple to implement, and has unique self-calibration features and low susceptibility to noise and cross-channel errors (Raney, 2007). In comparison to quad-pol, RCM CP mode can acquire a higher frequency of larger swaths $(350 \mathrm{~km})$, with medium spatial resolution $(50 \mathrm{~m})$; thus, it has the potential to be a good alternative to single-, dualand quad-pol SAR measurements for ocean surface wind fields.

Under low to moderate wind speeds, C-band SAR ocean wind retrieval models are generally based on geophysical model functions (GMFs) (Stoffelen and Anderson 1997; Hersbach et al. 2007; Hersbach 2010) derived from vertical transmit, vertical receive (VV-pol) scatterometer measurements. These GMFs relate the normalized radar cross section (NRCS) to the radar incidence angle, wind speed, and relative wind direction, and they are widely used for SAR ocean surface wind speed retrieval (Lehner et al. 1998; Horstmann et al. 2003; Zhang et al. 2012). Moreover, a VV-pol GMF, called C_SARMOD2, has been proposed, based on RADARSAT-2 (RS-2) and Sentinel-1A $(S-1 A)$ SAR data and collocated buoy winds; statistical validations show comparable wind speed retrieval accuracy compared to existing CMOD GMFs (Lu et al. 2018). In polar regions, SAR images are routinely acquired in horizontal transmit, horizontal receive ( $\mathrm{HH}-\mathrm{pol})$ due to its higher sensitivity to sea ice than VV-pol. Since there were no similarly well-developed CMOD GMFs for HH-pol, the NRCS in HHpol had to be converted to $\mathrm{VV}$-pol, using various empirical and theoretical polarization ratio (PR) models, before being 
applied to wind speed retrieval (Thompson et al. 1998; Vachon and Dobson 2000; Elfouhaily 1996; Mouche et al. 2005; Johnsen et al. 2008; Zhang et al. 2011). However, this transformation inevitably introduces error into the SAR-retrieved wind speeds. To avoid this issue, an HH-pol GMF called CMODH (B. Zhang et al. 2019) has been recently developed for ocean surface wind speed retrieval, based on a large number of collocations between the C-band Envisat ASAR HH-pol NRCS and ASCAT winds, which directly relates the HH-pol NRCS to wind vectors and radar incidence angles. The capability of $\mathrm{CMODH}$ for wind speed retrieval has been comprehensively validated, and shown to be consistent with buoy winds (Lu et al. 2021).

Prior to the RCM launch, great efforts were devoted to investigating the feasibility of CP SAR ocean wind retrieval. CP parameters were simulated from $R S$-2 quad-pol images using a RCM simulator (Charbonneau et al. 2010), and used to examine the dependency of incidence angle and wind vector on these parameters (Geldsetzer et al. 2015). Thus, it was found that right circular transmit, right circular receive (RR-pol) backscatter $\left(\sigma_{\mathrm{RR}}^{0}\right)$ data show a primary dependency on wind speed, and are also dependent on both incidence angle and wind direction; however, an analytic function and associated coefficients were not presented. Previous studies have demonstrated that wind speed can be estimated from the $\sigma_{\mathrm{RR}}^{0}$ using a combination of CMOD5 and a linear model (Denbina and Collins 2016). Moreover, in terms of wind speed retrieval accuracy, right circular transmit, vertical receive (RV-pol) backscatter $\left(\sigma_{\mathrm{RV}}^{0}\right)$ was found to be comparable to VV-pol backscatter $\left(\sigma_{\mathrm{VV}}^{0}\right)$, with CMOD GMFs (Geldsetzer et al. 2019). A simple CP wind speed retrieval model has been proposed, relating RR-pol backscatter and the wind speed (Fang et al. 2019), which has been further refined by incorporating additional incidence angle dependency (G. Zhang et al. 2019). However, the wind direction dependency of $\sigma_{\mathrm{RR}}^{0}$ was ignored in these studies.

The existing VV-and HH-pol GMFs cannot be directly used to retrieve wind speed with $\mathrm{CP}$ data. Until now, no available GMFs can directly connect CP SAR-measured backscatters and ocean surface wind vectors. Moreover, the previous RRpol wind speed retrieval model does not take into account the wind direction dependency of $\sigma_{\mathrm{RR}}^{0}$. In this study, for the first time, we present CP GMFs relating right circular transmit, horizontal receive ( $\mathrm{RH}-$ pol), right circular transmit, vertical receive (RV-pol), right circular transmit, left circular receive (RL-pol), and right circular transmit, right circular receive (RR-pol) backscatters to incidence angles, wind speeds and wind directions, for ocean wind retrieval. The formulations and coefficients of these CP GMFs are also given. In section 2, the dataset is briefly described. Section 3 presents GMF development and validation results. Summary and conclusions are given in section 4 .

\section{Dataset}

In this study, $1734 R S$-2 SAR images acquired in fine quadpol (HH, HV, VH, VV) imaging mode are collected between October 2008 and March 2017. The major parameters of the quad-pol mode, such as incidence angles, spatial resolutions, swath width, and noise-equivalent sigma-zero (NESZ) can be found in B. Zhang et al. 2019 (Table 1). These SAR images are able to be used for simulating CP data because the quad-pol single look complex (SLC) product provides measurements of the scattering matrix elements. All the $R S$ - 2 quad-pol SAR images are collocated with 60 in situ National Data Buoy Center (NDBC) buoy measurements in the Gulf of Alaska, Bering Sea, Gulf of Mexico, and off the east and west coasts of the United States and Canada. The temporal and spatial windows for the collocation are restricted to $30 \mathrm{~min}$ and $1 \mathrm{~km}$, respectively. This approach results in 1594 collocated data pairs where each pair includes radar backscatters $\left(\sigma_{\mathrm{HH}}^{0}, \sigma_{\mathrm{HV}}^{0}, \sigma_{\mathrm{VH}}^{0}, \sigma_{\mathrm{VV}}^{0}\right)$, radar incidence angle, buoy-measured wind speed and wind direction. Buoy-measured wind speeds at different heights are converted to $10-\mathrm{m}$ equivalent neutral wind conditions using a simple logarithmic wind profile model (Mears et al. 2001), which is given by

$$
U_{10}=\left[\ln \left(10 / z_{0}\right) / \ln \left(H / z_{0}\right)\right] \times U_{H},
$$

where $U_{10}$ is the wind speed at 10 -m height, $U_{H}$ is the wind speed at the height of the anemometer, $H$ is the height of the anemometer, and $z_{0}$ is the roughness length, which is empirically determined with a value of $1.52 \times 10^{-4}$ (Peixoto and Oort 1992). In this dataset, the entire range of incidence angles, wind speeds, and wind directions are between $20^{\circ}$ and $49^{\circ}, 3$ and $20 \mathrm{~m} \mathrm{~s}^{-1}$, and $0^{\circ}$ and $360^{\circ}$, respectively. This dataset is used to simulate $\mathrm{CP}$ data and then to develop and validate CP GMFs for wind speed retrieval.

\section{Model development and validation}

Under the assumption of scattering reciprocity (Nghiem et al. 1992), $S_{\mathrm{HV}}$ and $S_{\mathrm{VH}}$ are equal. Thus, the scattering vector elements for two CP modes (Nord et al. 2009) can be estimated from combinations of the elements of the quad-pol scattering matrix:

$$
\begin{aligned}
& S_{\mathrm{RH}}=\frac{1}{\sqrt{2}}\left(S_{\mathrm{HH}}-i S_{\mathrm{HV}}\right), \\
& S_{\mathrm{RV}}=\frac{1}{\sqrt{2}}\left(-i S_{\mathrm{VV}}+S_{\mathrm{HV}}\right), \\
& S_{\mathrm{RL}}=\frac{1}{2} i\left(S_{\mathrm{HH}}+S_{\mathrm{VV}}\right), \\
& S_{\mathrm{RR}}=\frac{1}{2}\left(S_{\mathrm{HH}}-S_{\mathrm{VV}}+i 2 S_{\mathrm{HV}}\right),
\end{aligned}
$$

where $S_{\mathrm{RH}}$ and $S_{\mathrm{RV}}$ are associated with right circular transmit, linear (horizontal and vertical) receive (CTLR) mode, and $S_{\mathrm{RL}}$ and $S_{\mathrm{RR}}$ correspond to right circular transmit, left or right circular receive, namely, dual-circular-polarimetric (DCP) mode. The quad-pol SAR SLC data are first used to estimate CP polarimetric scattering coefficients $\left(S_{\mathrm{RH}}, S_{\mathrm{RV}}, S_{\mathrm{RL}}, S_{\mathrm{RR}}\right)$ based on Eqs. (1)-(4). Subsequently, the simulated CP backscatters $\left(\sigma_{\mathrm{RH}}^{0}, \sigma_{\mathrm{RV}}^{0}, \sigma_{\mathrm{RL}}^{0}, \sigma_{\mathrm{RR}}^{0}\right)$ are obtained by estimating the product of polarimetric scattering coefficients and their conjugate values (e.g., $\sigma_{\mathrm{RH}}^{0}=S_{\mathrm{RH}} S_{\mathrm{RH}}^{*}$ ), which are given as follows:

$$
\sigma_{\mathrm{RH}}^{0}=\frac{1}{2}\left[\sigma_{\mathrm{HH}}^{0}+\sigma_{\mathrm{HV}}^{0}-2 \Re\left(S_{\mathrm{HH}} \cdot S_{\mathrm{HV}}^{*}\right)\right],
$$


TABLE 1. The specifications of the three RCM CP beam modes.

\begin{tabular}{lrcc}
\hline \multicolumn{1}{c}{ RCM beam mode } & $\begin{array}{c}\text { Resolution } \\
(\mathrm{m})\end{array}$ & $\begin{array}{c}\text { Swath } \\
\text { width }(\mathrm{km})\end{array}$ & $\begin{array}{c}\text { Nominal } \\
\text { NESZ }(\mathrm{dB})\end{array}$ \\
\hline Low-resolution $100 \mathrm{~m}$ & 100 & 500 & -22 \\
Low noise & 100 & 350 & -25 \\
Medium-resolution $50 \mathrm{~m}$ & 50 & 350 & -22 \\
\hline
\end{tabular}

$$
\begin{aligned}
\sigma_{\mathrm{RV}}^{0}= & \frac{1}{2}\left[\sigma_{\mathrm{VV}}^{0}+\sigma_{\mathrm{HV}}^{0}-2 \Re\left(S_{\mathrm{VV}} \cdot S_{\mathrm{HV}}^{*}\right)\right], \\
\sigma_{\mathrm{RL}}^{0}= & \frac{1}{4}\left[\sigma_{\mathrm{HH}}^{0}+\sigma_{\mathrm{VV}}^{0}+2 \Re\left(S_{\mathrm{HH}} \cdot S_{\mathrm{VV}}^{*}\right)\right], \\
\sigma_{\mathrm{RR}}^{0}= & \frac{1}{4}\left\{\sigma_{\mathrm{HH}}^{0}+\sigma_{\mathrm{VV}}^{0}+4 \sigma_{\mathrm{HV}}^{0}-2 \Re\left(S_{\mathrm{HH}} \cdot S_{\mathrm{VV}}^{*}\right)\right. \\
& \left.+4 \Im\left[\left(S_{\mathrm{HH}}-S_{\mathrm{VV}}\right) \cdot S_{\mathrm{HV}}^{*}\right]\right\},
\end{aligned}
$$

where $\sigma_{\mathrm{HH}}^{0}, \sigma_{\mathrm{VV}}^{0}$, and $\sigma_{\mathrm{HV}}^{0}$ are HH-, VV-, HV-pol radar backscatters, and $\Re$ and $\mathfrak{J}$ represent the real and the imaginary parts, respectively. There are three RCM imaging modes that are preferable for ocean wind mapping, which are low-resolution mode, low-noise mode, and medium-resolution mode. The specifications for these modes are given in Table 1, including spatial resolution, swath width and nominal NESZ. For low noise mode, NESZ is $-25 \mathrm{~dB}$. According to cross-pol wind speed retrieval models (Vachon and Wolfe 2011; Zhang and Perrie 2012), this NESZ corresponds to a wind speed of around $18 \mathrm{~m} \mathrm{~s}^{-1}$. Thus, when wind speeds are smaller than $18 \mathrm{~m} \mathrm{~s}^{-1}$, cross-pol radar returns are below this NESZ. Under these circumstances, $\sigma_{\mathrm{HV}}^{0}$ in (5) and (6) can be neglected. However, $\sigma_{\mathrm{HV}}^{0}$ in (8) cannot be ignored because the value of $4 \sigma_{\mathrm{HV}}^{0}$ is comparable to that of $\sigma_{\mathrm{HH}}^{0}$ or $\sigma_{\mathrm{VV}}^{0}$. Based on the reflection symmetry (Nghiem et al. 1992), the correlation between copol and cross-pol channels is zero; $S_{\mathrm{HH}} \cdot S_{\mathrm{HV}}^{*}=S_{\mathrm{VV}} \cdot S_{\mathrm{HV}}^{*}=0$. Thus, (5)-(8) are approximated as

$$
\begin{aligned}
\sigma_{\mathrm{RH}}^{0} & =\frac{1}{2} \sigma_{\mathrm{HH}}^{0}, \\
\sigma_{\mathrm{RV}}^{0} & =\frac{1}{2} \sigma_{\mathrm{VV}}^{0}, \\
\sigma_{\mathrm{RL}}^{0} & =\frac{1}{4}\left[\sigma_{\mathrm{HH}}^{0}+\sigma_{\mathrm{VV}}^{0}+2 \Re\left(S_{\mathrm{HH}} \cdot S_{\mathrm{VV}}^{*}\right)\right],
\end{aligned}
$$

$$
\sigma_{\mathrm{RR}}^{0}=\frac{1}{4}\left[\sigma_{\mathrm{HH}}^{0}+\sigma_{\mathrm{VV}}^{0}+4 \sigma_{\mathrm{HV}}^{0}-2 \Re\left(S_{\mathrm{HH}} \cdot S_{\mathrm{VV}}^{*}\right)\right],
$$

where $\sigma_{\mathrm{HH}}^{0}, \sigma_{\mathrm{VV}}^{0}$, and $\sigma_{\mathrm{HV}}^{0}$ can be estimated with $\mathrm{HH}-, \mathrm{VV}-$, and HV-pol GMFs, such as CMODH (B. Zhang et al. 2019), CMOD5.N (Hersbach 2010), and C-2PO (Zhang and Perrie 2012). Thus, (9)-(12) can be further expressed as

$$
\begin{aligned}
\sigma_{\mathrm{RH}}^{0} \approx & \frac{1}{2} \mathrm{CMODH}, \\
\sigma_{\mathrm{RV}}^{0} \approx & \frac{1}{2} \mathrm{CMOD} 5 . \mathrm{N}, \\
\sigma_{\mathrm{RL}}^{0} \approx & \frac{1}{4}(\mathrm{CMODH}+\mathrm{CMOD} 5 . \mathrm{N} \\
& +2 \sqrt{\mathrm{CMODH} \cdot \mathrm{CMOD} 5 \cdot \mathrm{N}}), \\
\sigma_{\mathrm{RR}}^{0} \approx & \frac{1}{4}(\mathrm{CMODH}+\mathrm{CMOD} 5 . \mathrm{N}+4 \mathrm{C} 2 \mathrm{PO} \\
& -2 \sqrt{\mathrm{CMODH} \cdot \mathrm{CMOD} 5 \cdot \mathrm{N}}) .
\end{aligned}
$$

Before developing CP GMFs for wind speed retrieval, we need to first examine the relation between RH-, RV-, RL- and RRpol radar backscatters and incidence angles, wind speeds, and relative wind directions. Figure 1a clearly shows that $\sigma_{\mathrm{RH}}^{0}, \sigma_{\mathrm{RV}}^{0}$, and $\sigma_{\mathrm{RL}}^{0}$ rapidly decrease with increasing incidence angles. They also exhibit a certain modulation with respect to wind directions and show dependency of wind speed, as exhibited in Figs. $1 \mathrm{~b}$ and $1 \mathrm{c}$. By contrast, $\sigma_{\mathrm{RR}}^{0}$ is less sensitive to radar incidence angle, especially for large incidence angles, and also to wind direction. Moreover, $\sigma_{\mathrm{RR}}^{0}$ significantly depends on wind speed.

According to the above analysis, we relate $\mathrm{CP}$ radar backscatters to incidence angle, wind speed, and relative wind direction based on the CMODH framework (B. Zhang et al. 2019), which is given as follows:

$$
\begin{aligned}
\sigma_{t r}^{0}(\nu, \phi, \theta)= & \left\{B _ { 0 } ( \nu , \theta ) \left[1+B_{1}(\nu, \theta) \cos (\phi)\right.\right. \\
& \left.\left.+B_{2}(\nu, \theta) \cos (2 \phi)\right]\right\}^{p},
\end{aligned}
$$

where $t$ and $r$ represent the transmitting and receiving polarizations (e.g., RH or RV, or RL or RR), respectively; $B_{0}$ is an isotropic quantity; and $B_{1}$ and $B_{2}$ describe the
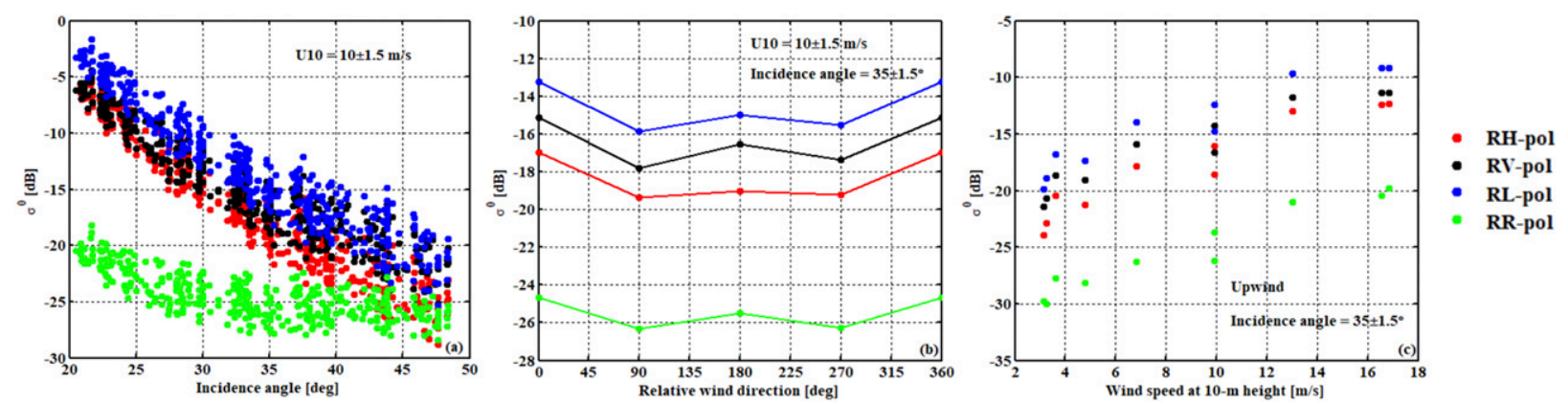

FIG. 1. Simulated RH-, RV-, RL- and RV-pol NRCS from $R S$-2 quad-pol SAR SLC images vs (a) incidence angle, (b) relative wind direction, and (c) wind speed. 

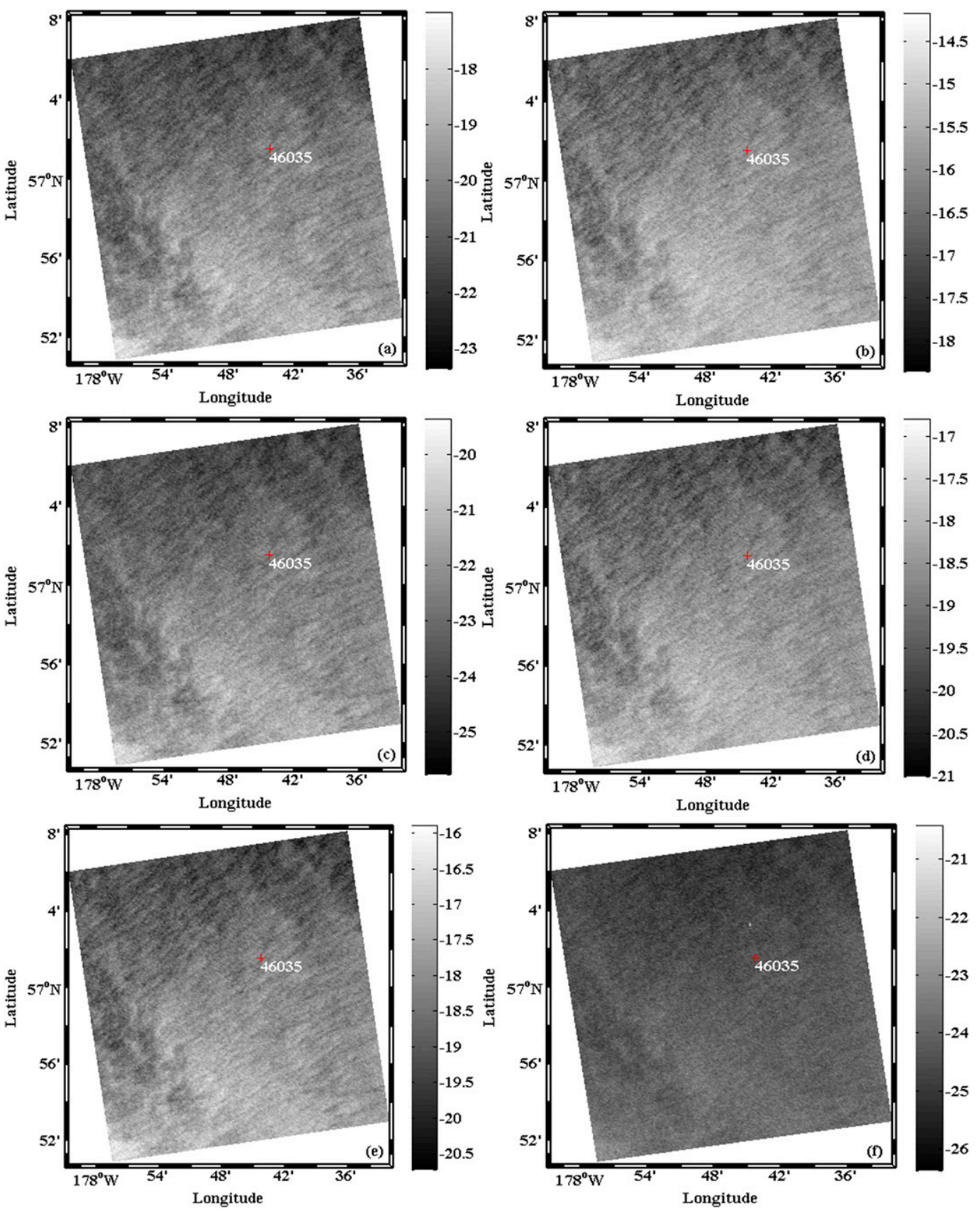

FIG. 2. C-band (a) HH-pol and (b) VV-pol $R S$-2 SAR images acquired in the fine quad-polarization mode at 0545 UTC 23 Oct 2011 (the grayscale color bar denotes $\sigma_{0}$, measured in decibels). Simulated (c) RH-pol, (d) RVpol, (e) RL-pol, and (f) RR-pol SAR images are from quad-pol data as shown above. The location of the NDBC buoy (46035) is indicated by the red plus sign (+). The RADARSAT-2 data are a product of MacDonald, Dettwiler, and Associates, Ltd. All rights reserved.

upwind-downwind and the upwind-crosswind amplitudes. All of these are functions of wind speed $\nu$ and incidence angle $\theta$. The superscript $p$ is a constant with a value of 1.6. The superscript $p$ and the transfer functions used to define $B_{0}, B_{1}$, and $B_{2}$ are adopted from CMODH (B. Zhang et al. 2019) for use in this study.

We randomly select 798 collocated data pairs to tune the proposed CP GMFs (CMODRH, CMODRV, CMODRL, and
CMODRR) and derive their coefficients. The remaining 796 matchup data pairs are used for the validation of these GMFs, as an independent dataset. $B_{i}(i=0,1,2)$ coefficients are determined via a nonlinear regression analysis approach, by minimizing the standard deviation between the $\mathrm{CP}$ radar backscatter estimates and regression analysis for all wind speeds, wind directions and incidence angles. The final model formulation and 

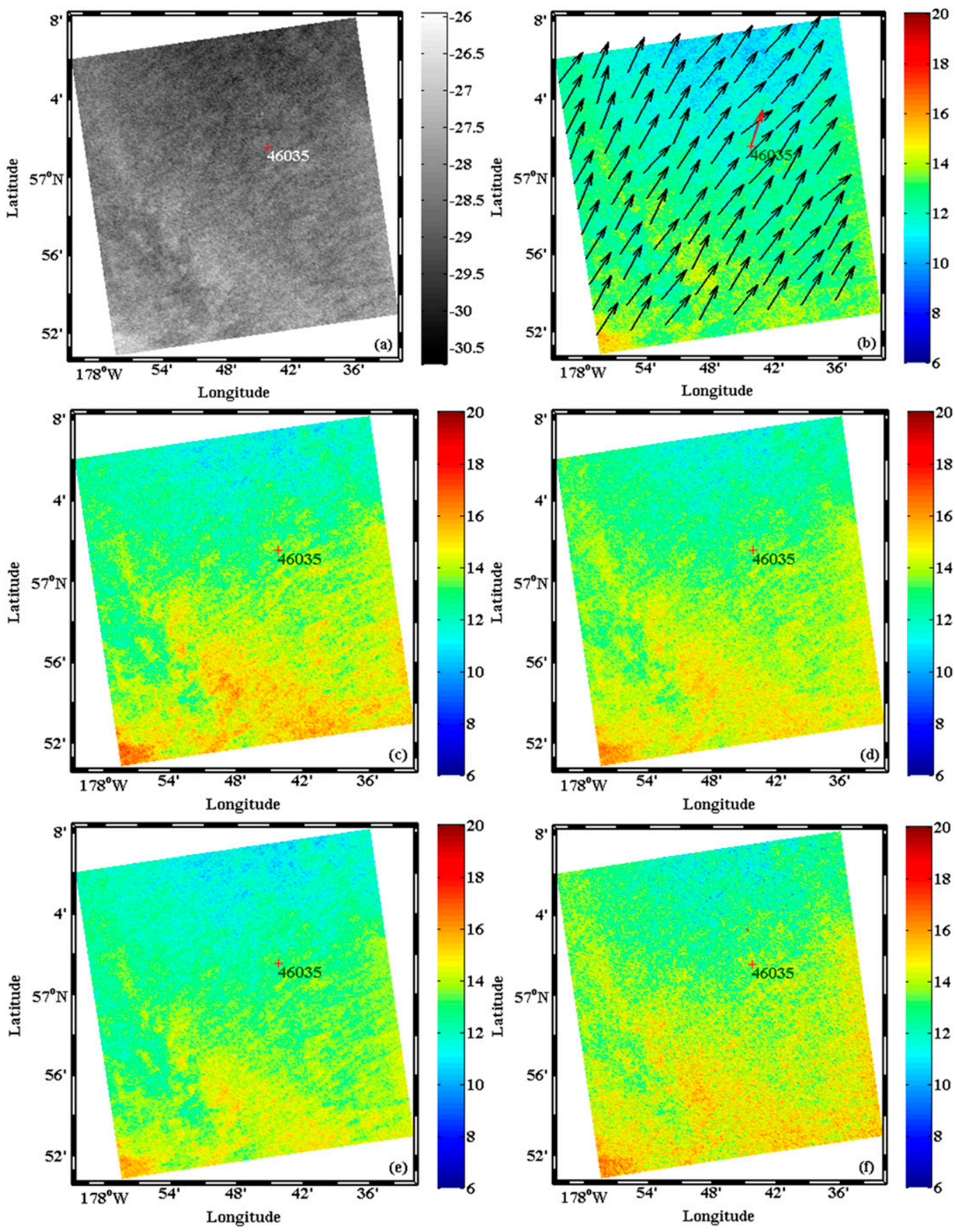

FIG. 3. (a) C-band HV-pol $R S-2$ SAR image acquired in the fine quad-polarization mode at 0545 UTC 23 Oct 2011 (the grayscale color bar denotes $\sigma_{0}$, measured in decibels). (b) SAR-retrieved wind speeds using the C-2PO model and HV-pol SAR image with overlaid wind directions. SAR-retrieved wind speeds with proposed CP GMFs: (c) CMODRH, (d) CMODRV, (e) CMODRL, and (f) CMODRR. The color bar denotes the wind speed ( $\mathrm{m} \mathrm{s}^{-1}$ ). The NDBC buoy (46035) is indicated by the red plus sign $(+)$.

28 coefficients for RH-, RV-, RL-, and RR-pol are given in the appendix. In this study, it should be noted that all models are developed from the same dataset. To assess the accuracy of $\mathrm{CP}$ GMFs, we carry out both case studies and a statistical validation, using the independent dataset as mentioned above.

\section{a. Case validation}

Figures $2 \mathrm{a}$ and $2 \mathrm{~b}$ illustrate the HH- and VV-pol NRCS from an $R S$-2 fine quad-pol SAR image acquired at 0545 UTC 23 October 2011. The HV-pol NRCS is shown in Fig. 3a. The original pixel spacing of the fine quad-pol SAR image is $5 \mathrm{~m}$. 

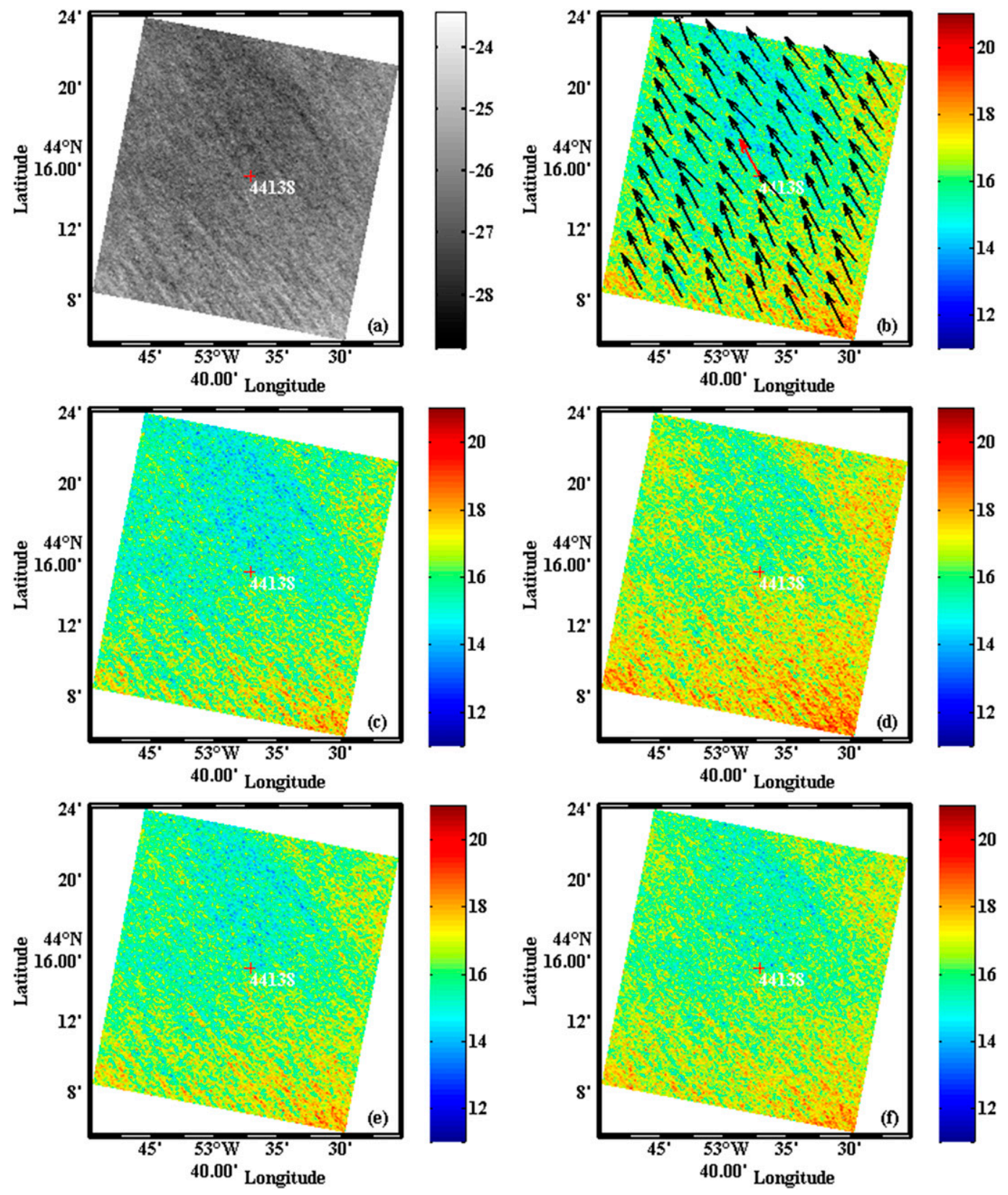

FIG. 4. (a) C-band HV-pol $R S$-2 SAR image acquired in the fine quad-polarization mode at 0946 UTC 16 Dec 2009 (the grayscale color bar denotes $\sigma_{0}$, measured in decibels). (b) SAR-retrieved wind speeds using the C-2PO model and HV-pol SAR image with overlaid wind directions. SAR-retrieved wind speeds with proposed CP GMFs: (c) CMODRH, (d) CMODRV, (e) CMODRL, and (f) CMODRR. The color bar denotes the wind speed ( $\left.\mathrm{m} \mathrm{s}^{-1}\right)$. The NDBC buoy (44138) is indicated by the red plus sign $(+)$.

We apply the $20 \times 20$ pixel boxcar averaging to the NRCS to eliminate speckle noise. Thus, the resampled pixel spacing (RPS) is $100 \mathrm{~m}$. This SAR image is collocated with a NDBC buoy $\left(46035,57^{\circ} 1^{\prime} 33^{\prime \prime} \mathrm{N}, 177^{\circ} 44^{\prime} 16^{\prime \prime} \mathrm{W}\right)$ in the Bering Sea. We use the quad-pol SAR image to simulate the RH-, RV-, RLand RR-pol SAR data, which are shown in Figs. 2c-f. These simulated CP SAR images are used to retrieve wind speeds with CMODRH, CMODRV, CMODRL, and CMODRR.
Since there are two unknown parameters, wind speed and wind direction, that exist in these GMFs, wind directions have to be determined before wind speed can be retrieved. Here, the C-band cross-pol wind speed retrieval model (C-2PO) (Zhang and Perrie 2012) and HV-pol NRCS are first used to derive wind speed. Subsequently, the VV-pol NRCS and radar incidence angles, along with the retrieved wind speeds, are substituted into CMOD5.N (Hersbach 2010), with the result 

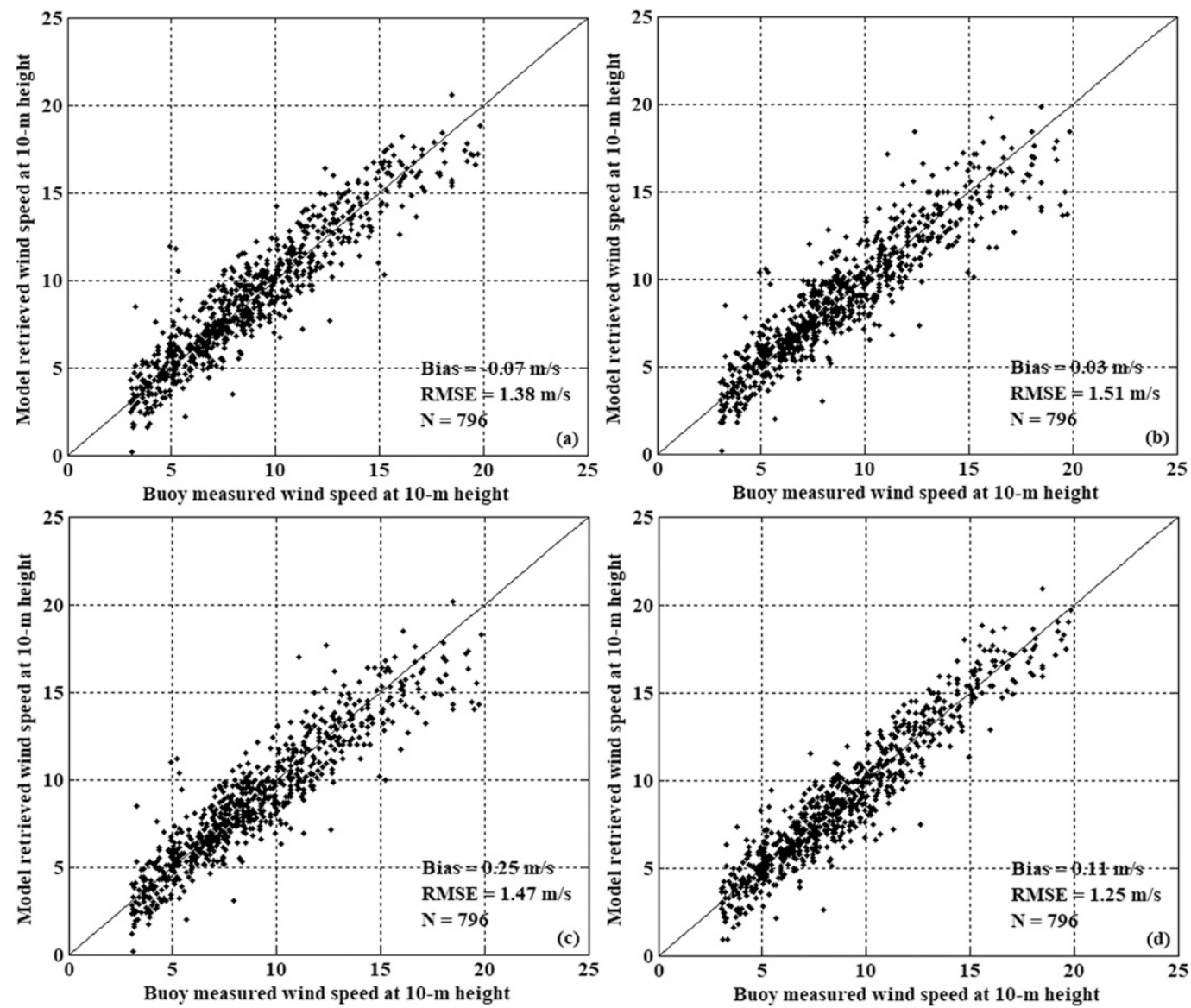

FIG. 5. Comparisons of SAR-retrieved wind speeds and in situ buoy wind speeds using simulated RH-, RV-, RL-, and RR-pol SAR data from $R S-2$ quad-pol SAR SLC images and the proposed CP GMFs: (a) CMODRH, (b) CMODRV, (c) CMODRL, and (d) CMODRR.

that the wind directions have $180^{\circ}$ ambiguities. Finally, we use buoy-measured wind directions to remove the wind direction ambiguities. Figure $3 \mathrm{~b}$ shows wind speeds derived from $\mathrm{C}-2 \mathrm{PO}$ model, overlaid with estimated wind directions. The buoy wind direction is $198^{\circ}$, whereas the SAR-retrieved wind direction at the buoy site is $216^{\circ}$. Given the wind directions, incidence angles, RH-, RV-, RL-, and RR-pol NRCS, we can use CMODRH, CMODRV, CMODRL, and CMODRR to directly retrieve wind speeds. The retrieved wind speeds are shown in Figs. 3c-f. The buoy-measured wind speed at $10-\mathrm{m}$ height is $12.8 \mathrm{~m} \mathrm{~s}^{-1}$, whereas the wind speeds inferred by CMODRH, CMODRV, CMODRL, and CMODRR are 13.4, 13.3, 12.9, and $13.5 \mathrm{~m} \mathrm{~s}^{-1}$, respectively. Figure 4 shows another case of CP SAR wind retrieval. Wind streaks can be clearly found in the bottom of HV-pol SAR image, as shown in Fig. 4a. There is a buoy maintained by Environment and Climate Change Canada
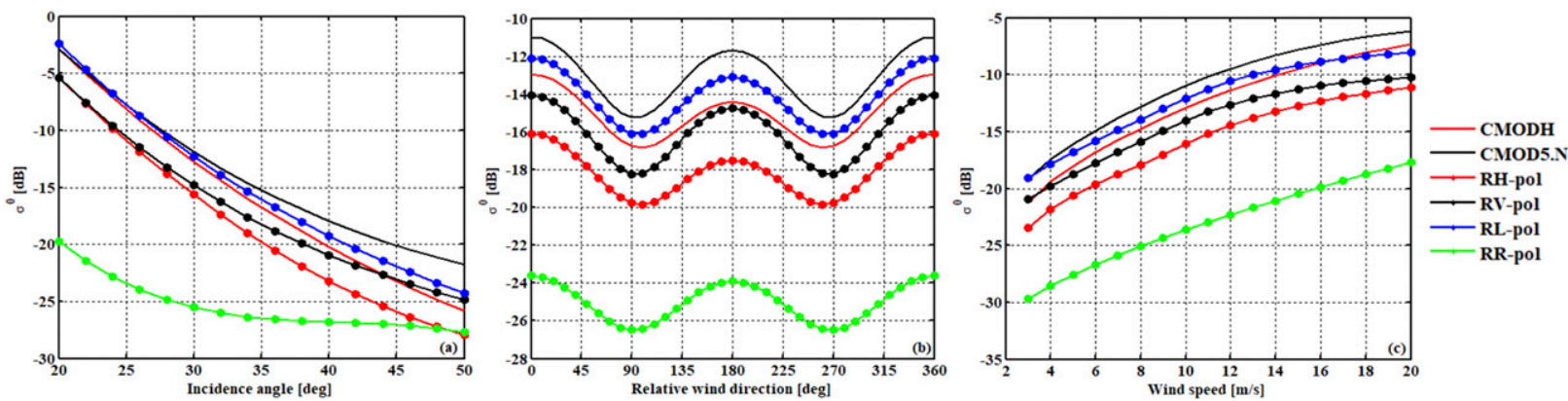

FIG. 6. Estimated HH-, VV-, RH-, RV-, RL-, and RR-pol NRCS from HH- and VV-pol GMFs and CP GMFs vs (a) incidence angles $\left(\phi=0^{\circ}, \nu=10 \mathrm{~m} \mathrm{~s}^{-1}\right),(\mathrm{b})$ relative wind directions $\left(\theta=35^{\circ}, \nu=10 \mathrm{~m} \mathrm{~s}^{-1}\right)$, and (c) wind speeds $\left(\phi=0^{\circ}, \theta=35^{\circ}\right)$. 


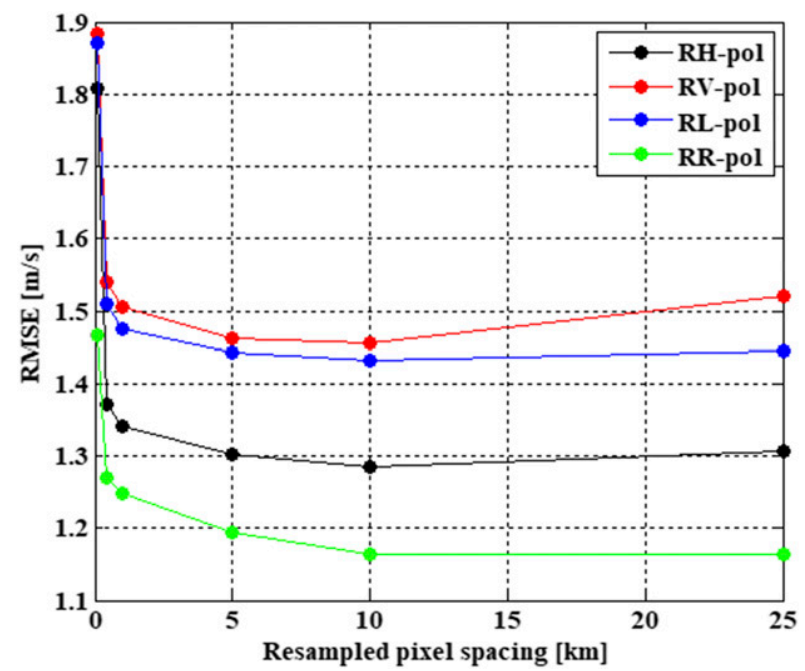

FIG. 7. The RMSE between SAR-retrieved wind speeds using the proposed CP GMFs and in situ buoy wind speeds vs different resampled pixel spacings $(100,400,1000,5000,10000$, and $25000 \mathrm{~m})$.

$\left(44138,44^{\circ} 15^{\prime} 0^{\prime \prime} \mathrm{N}, 53^{\circ} 37^{\prime} 48^{\prime \prime} \mathrm{W}\right)$, located in this SAR imaging area. Figure $4 \mathrm{~b}$ illustrates SAR-retrieved wind speeds using the C-2PO model and HV-pol SAR image with overlaid wind directions. The retrieved wind speeds from CMODRH, CMODRV, CMODRL, and CMODRR models are shown in Figs. 4c-f. For this case, the buoy-measured wind speed at $10-\mathrm{m}$ height is $15.5 \mathrm{~m} \mathrm{~s}^{-1}$, whereas the wind speeds inferred by CMODRH, CMODRV, CMODRL, and CMODRR are 15.5, $16.0,15.5$, and $16.0 \mathrm{~m} \mathrm{~s}^{-1}$, respectively.

\section{b. Statistical validation}

In addition to case study validation, we also carry out a statistical comparison between wind speed retrievals and buoy measurements. As mentioned above, almost $50 \%$ of collocated data pairs are used as an independent dataset for evaluating the performance of the proposed CP GMFs. Figure 5 shows a set of plots of the retrieved wind speeds against the buoy measurements. It should be noted that the wind direction inputs for the CP GMFs are obtained from the buoy measurements. As shown in Figs. $5 b$ and 5c, CMODRV and CMODRL have similar wind speed retrieval accuracies, with RMSE values of $\sim 1.5 \mathrm{~m} \mathrm{~s}^{-1}$. CMODRL shows significant underestimates for wind speeds, ranging between 15 and $20 \mathrm{~m} \mathrm{~s}^{-1}$. As demonstrated in Lu et al. (2021), the wind speed retrieval capability of CMODH is better than that of CMOD5.N for incidence angles between $30^{\circ}$ and $49^{\circ}$ and wind speeds between 10 and $20 \mathrm{~m} \mathrm{~s}^{-1}$. In our study, we suggest that this conclusion also holds for CMODRH. Compared to CMODRV and CMODRL, CMODRH has smaller bias and RMSE values of 0.07 and $1.38 \mathrm{~m} \mathrm{~s}^{-1}$, respectively. The combination of RH- and RV-pol SAR images has potential to improve wind speed retrieval. Among all the GMFs, CMODRR-retrieved wind speeds are closest to buoy measurements, corresponding to the smallest RMSE of $1.25 \mathrm{~m} \mathrm{~s}^{-1}$. Figure $5 \mathrm{~d}$ shows that CMODRR significantly improves wind speed retrieval, especially for wind speeds ranging from 15 to $20 \mathrm{~m} \mathrm{~s}^{-1}$. This is possibly due to the fact that $\sigma_{\mathrm{RR}}^{0}$ is less sensitive to incidence angles and wind directions, as shown in Figs. 1a and 1b. Previous studies have reported that $\sigma_{\mathrm{RR}}^{0}$ is more dependent on wind speed than $\sigma_{\mathrm{RH}}^{0}, \sigma_{\mathrm{RV}}^{0}$, and $\sigma_{\mathrm{RL}}^{0}$ (Geldsetzer et al. 2015; G. Zhang et al. 2019); thus, we suggest that $\sigma_{\mathrm{RR}}^{0}$ is appropriate for high wind speed retrieval.

VV- and HH-pol SAR imagery are routinely used for ocean surface wind speed retrieval for low to moderate winds (Lehner et al. 1998; Horstmann et al. 2003; Zhang et al. 2012; Lu et al. 2018; B. Zhang et al. 2019; Lu et al. 2021). According to Eqs. (5)-(7), CP radar backscatters are a combination of radar returns from co- and cross-pol channels. Under high wind conditions, VV-pol radar backscatters begin to saturate which leads to wind speed underestimation. Thus, this possibly accounts for the fact that wind speeds retrieved from RH-, RV-, and RL-pol GMFs are smaller than buoy measurements when wind speeds are between 18 and $20 \mathrm{~m} \mathrm{~s}^{-1}$ as shown in Figs. 5ac. By contrast, VH-pol radar backscatters have not shown any sign of saturation under high wind conditions and have therefore been widely used for hurricane wind speed retrieval (Zhang and Perrie 2012; Zhang et al. 2014; Mouche et al. 2017). $\sigma_{\mathrm{RR}}^{0}$ includes an important HV-pol contribution [e.g., Eq. (8)] and shows prominent wind speed dependency like $\sigma_{\mathrm{HV}}^{0}$ (see Fig. 1d). Thus, Fig. 5d shows that RR-pol GMF-retrieved wind speeds are closer to buoy data compared to those from RH-, RV- and RL-pol GMFs, especially for high winds.

By using the proposed CP GMFs, CMODH (B. Zhang et al. 2019) and CMOD5.N (Hersbach 2010), we can now analyze the dependence of incidence angle, wind speed and wind direction on HH-, VV-, RH-, RV-, RL-, and RR-pol radar backscatters. As shown in Fig. 6, the variations and trends of $\sigma_{\mathrm{RH}}^{0}$ and $\sigma_{\mathrm{RV}}^{0}$ are similar to those of $\sigma_{\mathrm{HH}}^{0}$ and $\sigma_{\mathrm{VV}}^{0}$. CMODRLestimated $\sigma_{\mathrm{RL}}^{0}$ values are about the averages of $\sigma_{\mathrm{HH}}^{0}$ and $\sigma_{\mathrm{VV}}^{0}$, which indicates that the approximation given in (15) is reasonable. However, $\sigma_{\mathrm{RR}}^{0}$ exhibits distinctive incidence angle, wind speed and wind direction dependencies, which are different from those of $\sigma_{\mathrm{HH}}^{0}, \sigma_{\mathrm{VV}}^{0}, \sigma_{\mathrm{RH}}^{0}, \sigma_{\mathrm{RV}}^{0}$, and $\sigma_{\mathrm{RL}}^{0}$. For upwind conditions, and a wind speed of $10 \mathrm{~m} \mathrm{~s}^{-1}, \sigma_{\mathrm{RR}}^{0}$ decreases with increasing incidence angles. The range of variability is between 1 and $5 \mathrm{~dB}$ when incidence angles are between $20^{\circ}$ and $40^{\circ}$. Figure 6a clearly illustrates that $\sigma_{\mathrm{RR}}^{0}$ becomes insensitive to incidence angles when incidence angles are larger than $40^{\circ}$. Moreover, $\sigma_{R R}^{0}$ is not sensitive to wind direction but significantly depends on wind speed, as shown in Figs. 6b and 6c. According to (12), $\sigma_{\mathrm{RR}}^{0}$ includes the important HV-pol contribution, which is comparable to $\mathrm{HH}$ - or VV-pol, and thus shows prominent wind speed dependency like $\sigma_{\mathrm{HV}}^{0}$ (Zhang and Perrie 2012). We suggest that CP mode (right circular transmit, right circular receive) SAR images acquired with medium resolution $(50 \mathrm{~m})$ and wide swath $(350 \mathrm{~km})$ are appropriate for ocean surface wind speed measurements under high wind conditions, especially for tropical cyclones.

We carried out a numerical experiment to analyze the impact of spatial resolution on CP SAR wind speed retrieval accuracy. The RMSE of wind speed retrieval decreases with increasing RPS, and begins to level off when RPS is larger than $10 \mathrm{~km}$ (Fig. 7). Values for RMSE of RR-pol are always smaller than those of RH-, RV-, and RL-pol, whatever the RPS. In this 
TABLE A1. The coefficients of compact polarimetric geophysical model functions (CMODRH, CMODRV, CMODRL, and CMODRR).

\begin{tabular}{|c|c|c|c|c|c|}
\hline Function & Coefficients & $\mathrm{RH}$ & RV & RL & RR \\
\hline \multirow{13}{*}{$B_{0}$} & $c_{1}$ & 0.4936761895 & -0.9905120469 & -0.9540313467 & 3.7106196334 \\
\hline & $c_{2}$ & -3.1299521212 & -1.6469686282 & -1.8225793037 & 1.5769591619 \\
\hline & $c_{3}$ & 0.1294616791 & 0.7830322310 & 0.7710022832 & -1.0245537500 \\
\hline & $c_{4}$ & 0.0515827505 & -0.5651087876 & -0.6081363657 & -0.7781839751 \\
\hline & $c_{5}$ & -0.0167179443 & 0.0109808868 & 0.0148198445 & -0.0111882042 \\
\hline & $c_{6}$ & 0.0559713579 & 0.0469035902 & 0.0516234306 & 0.0489181545 \\
\hline & $c_{7}$ & 0.0498121092 & 0.1406372324 & 0.1419850430 & 0.0128772980 \\
\hline & $c_{8}$ & 0.0159907638 & 0.1211123349 & 0.1228889345 & -0.0235318067 \\
\hline & $c_{9}$ & 8.0358118309 & 2.7805258913 & 2.6510035016 & 18.9261442021 \\
\hline & $c_{10}$ & -7.0509932951 & -2.6196253127 & -2.4741606673 & 4.5928143938 \\
\hline & $c_{11}$ & -2.4988330960 & 0.7548687481 & 1.2007215175 & -4.7808595529 \\
\hline & $c_{12}$ & 0.2641939794 & 0.4366689978 & 0.4809730490 & -0.0669888987 \\
\hline & $c_{13}$ & 0.0252494889 & 0.0735632858 & 0.1114837190 & 0.0089466449 \\
\hline \multirow[t]{5}{*}{$B_{1}$} & $c_{14}$ & 0.1122148140 & 0.0447570559 & 0.0769799706 & 0.0076343062 \\
\hline & $c_{15}$ & 0.0091699097 & 0.0066345637 & 0.0079262188 & 0.0056794601 \\
\hline & $c_{16}$ & 0.0545113796 & 0.1769847686 & 0.1195093539 & -0.0505364701 \\
\hline & $c_{17}$ & 0.0382121887 & 0.0266227608 & 0.0322103927 & 0.0438169982 \\
\hline & $c_{18}$ & 17.9440232471 & 19.0136522543 & 18.0992834092 & 54.5467593743 \\
\hline \multirow[t]{10}{*}{$B_{2}$} & $c_{19}$ & 3.0684160071 & 2.8533213574 & 2.8727718171 & 1.1639252234 \\
\hline & $c_{20}$ & 12.6144918240 & 6.9500270356 & 8.3752001309 & -0.6020615735 \\
\hline & $c_{21}$ & 4.5305635266 & 4.8704629783 & 4.8944050554 & 5.3193249741 \\
\hline & $c_{22}$ & -1.6822642578 & -2.2862035239 & -2.3499197999 & -6.1465030728 \\
\hline & $c_{23}$ & 0.3358097597 & -0.3990879721 & -0.7594183156 & 6.5318830099 \\
\hline & $c_{24}$ & 25.5701814766 & 19.5955625768 & 19.7172544095 & 4.3765338740 \\
\hline & $c_{25}$ & 7.5305013854 & 9.1986614374 & 7.2927373291 & 7.8773375959 \\
\hline & $c_{26}$ & 5.4322221922 & 3.2281793349 & 3.3367861282 & 3.6782878071 \\
\hline & $c_{27}$ & 9.8384186774 & 8.5955358705 & 8.4826273317 & 2.6853416546 \\
\hline & $c_{28}$ & 2.0947582451 & 3.5945362039 & 2.7954955998 & 2.8250700820 \\
\hline
\end{tabular}

study, the pixel spacing of CP SAR images is resampled to $1 \mathrm{~km}$ for the purpose of noise removal, before developing the proposed CP GMFs. For CP SAR images acquired with wide swath $(350 \mathrm{~km})$ and medium $(50$ or $100 \mathrm{~m})$ resolution, $1 \mathrm{~km}$ resolution for wind speed is appropriate for operational SAR wind mapping.

\section{Summary and conclusions}

$\mathrm{CP}$ radar backscatters are examined for their dependencies as a function of incidence angle, wind speed and wind direction. We find that RH-, RV-, and RL-pol radar returns have similar incidence angle and wind vector dependencies as those of $\mathrm{HH}$ or VV-pol, whereas RR-pol signals are not sensitive to incidence angle or wind directions but significantly depend on wind speed. Thus, RR-pol measurements are expected to have good performance for high wind speed retrievals.

We develop CP GMFs for RCM wind speed retrieval, called CMODRH, CMODRV, CMODRL, and CMODRR, which respectively relate $\mathrm{RH}-, \mathrm{RV}-, \mathrm{RL}-$, and $\mathrm{RR}$-pol radar backscatters to incidence angles, wind speeds and wind directions. To validate the performance of these GMFs, we use these formulations to retrieve wind speeds and compare with buoy measurements. The wind speed retrieval accuracies of CMODRH, CMODRV, and CMODRL are comparable to those of CMODH and CMOD5.N, with RMSE values of $\sim 1.5 \mathrm{~m} \mathrm{~s}^{-1}$. Compared to the three CP GMFs mentioned above, CMODRR-retrieved wind speeds are closest to buoy measurements, with the smallest RMSE of $1.25 \mathrm{~m} \mathrm{~s}^{-1}$. In addition, CMODRR significantly improves wind speed retrieval accuracy when wind speeds are between 15 and $20 \mathrm{~m} \mathrm{~s}^{-1}$.

This study provides promising results for wind speed retrieval with the proposed CP GMFs and simulated CP data. CMODRH, CMODRV, and CMODRL are preferable for low and moderate wind speed retrieval, while CMODRR is appropriate for high winds, because RR-pol radar backscatters have weak wind direction and incidence angle dependency. These GMFs need to be further assessed and refined by using a large number of RCM SAR images acquired in CP imaging mode over meteorological buoys.

Acknowledgments. The authors thank the Canadian Space Agency for providing $R A D A R S A T-2 \mathrm{SAR}$ images. The NOAA buoy data are downloaded from http://www.ndbc.noaa.gov/. This work was supported in part by the National Science Foundation of China under Grant 42076181, in part by the NSFC-RSF Joint Project under Grant 42061134016; in part by the International Cooperation Project of National Science Fundation of China under Grant 41620104003; in part by the National Key Research and Development Program of China under Grant 2016YFC1401001; in part by the Key Project of Natural Science Research in Colleges and Universities under Grant 18KJA170002; by the Data Utilization Application Plan of the Canadian Space Agency, the Ocean Frontier Institute of Dalhousie University, Fisheries and Oceans Canada SWOT 
program, and the Postgraduate Research and Practice Innovation Program of Jiangsu Province under Grant 1344051901083, and the ESA-MOST China Dragon-5 Programme under Grant 58290.

\section{APPENDIX}

\section{CP GMF Formulation and Coefficients}

The form of the GMF is

$$
\begin{aligned}
\sigma_{t r}^{0}(\nu, \phi, \theta)= & \left\{B _ { 0 } ( \nu , \theta ) \left[1+B_{1}(\nu, \theta) \cos (\phi)\right.\right. \\
& \left.\left.+B_{2}(\nu, \theta) \cos (2 \phi)\right]\right\}^{p}
\end{aligned}
$$

where $t$ and $r$ represent the transmitting and receiving polarization, respectively; $p$ is constant with a value of 1.6 ; and $B_{0}, B_{1}$, and $B_{2}$ are functions of wind speeds $\nu$ and the incidence angle $\theta$, or alternatively, $x=(\theta-40) / 25$. The $B_{0}$ term is defined as

$$
B_{0}=10^{a_{0}+a_{1} \nu} f\left(a_{2} \nu, s_{0}\right)^{\gamma}
$$

where

$$
f\left(s, s_{0}\right)= \begin{cases}\left(s / s_{0}\right)^{\alpha} g\left(s_{0}\right), & s<s_{0} \\ g(s), & s \geq s_{0}\end{cases}
$$

where

$$
g(s)=1 /[1+\exp (-s)] \quad \text { and } \quad \alpha=s_{0}\left[1-g\left(s_{0}\right)\right] .
$$

The functions $a_{0}, a_{1}, a_{2}, \gamma$, and $s_{0}$ depend on incidence angle only:

$$
\begin{aligned}
& a_{0}=c_{1}+c_{2} x+c_{3} x^{2}+c_{4} x^{3}, \\
& a_{1}=c_{5}+c_{6} x, \\
& a_{2}=c_{7}+c_{8} x, \\
& \gamma=c_{9}+c_{10} x+c_{11} x^{2}, \\
& s_{0}=c_{12}+c_{13} x .
\end{aligned}
$$

The $B_{1}$ term is defined as follows:

$$
B_{1}=\frac{c_{14}(1+x)-c_{15} \nu\left\{0.5+x-\tanh \left[4\left(x+c_{16}+c_{17} \nu\right)\right]\right\}}{1+\exp \left[0.34\left(\nu-c_{18}\right)\right]} .
$$

The $B_{2}$ term is chosen as

$$
B_{2}=\left(-d_{1}+d_{2} \nu_{2}\right) \exp \left(-\nu_{2}\right)
$$

Here, $\nu_{2}$ is given by

$$
\nu_{2}=\left\{\begin{array}{ll}
a+b(y-1)^{n}, & y<y_{0} \\
y, & y \geq y_{0}
\end{array}, \quad y=\frac{\nu+\nu_{0}}{\nu_{0}},\right.
$$

where

$$
\begin{gathered}
y_{0}=c_{19}, \quad n=c_{20}, \\
a=y_{0}-\left(y_{0}-1\right) / n, \quad b=1 /\left[n\left(y_{0}-1\right)^{n-1}\right] .
\end{gathered}
$$

The quantities $\nu_{0}, d_{1}$, and $d_{2}$ are functions of incidence angle only:

$$
\begin{aligned}
& \nu_{0}=c_{21}+c_{22} x+c_{23} x^{2}, \\
& d_{1}=c_{24}+c_{25} x+c_{26} x^{2}, \\
& d_{2}=c_{27}+c_{28} x .
\end{aligned}
$$

The coefficients are given in Table A1.

\section{REFERENCES}

Bourassa, M. A., and Coauthors, 2019: Remotely sensed winds and wind stresses for marine forecasting and ocean modeling. Front. Mar. Sci., 6, 443, https://doi.org/10.3389/ fmars.2019.00443.

Charbonneau, F. J., and Coauthors, 2010: Compact polarimetry overview and applications assessment. Can. J. Remote Sens., 36, S298-S315, https://doi.org/10.5589/m10-062.

Chelton, D. B., and S. P. Xie, 2010: Coupled ocean-atmosphere interaction at oceanic mesoscales. Oceanography, 23 (4), $52-$ 69, https://doi.org/10.5670/oceanog.2010.05.

Denbina, M., and M. J. Collins, 2016: Wind speed estimation using C-band compact polarimetric SAR for wide swath imaging modes. ISPRS J. Photogramm. Remote Sens., 113, 75-85, https://doi.org/10.1016/j.isprsjprs.2016.01.002.

Elfouhaily, T., 1996: Physical modeling of electromagnetic backscatter from the ocean surface: Application to retrieval of wind fields and wind stress by remote sensing of the marine atmospheric boundary layer. Ph.D. dissertation, Université Paris VII.

Fang, H., and Coauthors, 2019: Ocean surface wind speed retrieval using simulated RADARSAT Constellation Mission compact polarimetry SAR data. Remote Sens., 11, 1876, https://doi.org/ 10.3390/rs11161876.

Geldsetzer, T., F. Charbonneau, M. Arkett, and T. Zagon, 2015: Ocean wind study using simulated RCM compact-polarimetry SAR. Can. J. Remote Sens., 41, 418-430, https://doi.org/ 10.1080/07038992.2015.1104635.

_ , S. K. Khurshid, W. Warner, F. Botelho, and D. Flett, 2019: Wind speed retrieval from simulated RADARSAT Constellation Mission compact polarimetry SAR data for marine application. Remote Sens., 11, 1682, https://doi.org/10.3390/ rs11141682.

Hersbach, H., 2010: Comparison of C-band scatterometer CMOD5.N equivalent neutral winds with ECMWF. J. Atmos. Oceanic Technol., 27, 721-736, https://doi.org/10.1175/2009JTECHO698.1.

—_, A. Stoffelen, and S. de Hann, 2007: An improved C-band scatterometer ocean geophysical model function: CMOD5. J. Geophys. Res., 112, C03006, https://doi.org/10.1029/ 2006JC003743.

Horstmann, J., H. Schiller, J. Schulz-Stellenfleth, and S. Lehner, 2003: Global wind speed retrieval from SAR. IEEE Trans. Geosci. Remote Sens., 41, 2277-2286, https://doi.org/10.1109/ TGRS.2003.814658.

Johnsen, H., G. Engen, and G. Guitton, 2008: Sea-surface polarization ratio from Envisat ASAR AP data. IEEE Trans. Geosci. Remote Sens., 46, 3637-3646, https://doi.org/10.1109/ TGRS.2008.2001061.

Lehner, S., J. Horstmann, W. Koch, and W. Rosenthal, 1998: Mesoscale wind measurements using recalibrated ERS SAR images. J. Geophys. Res., 103, 7847-7856, https://doi.org/ 10.1029/97JC02726.

Lu, Y., B. Zhang, W. Perrie, A. Mouche, X. Li, and H. Wang, 2018: A C-band geophysical model function for determining coastal wind speed using synthetic aperture radar. IEEE J. Sel. Top. 
Appl. Earth Obs. Remote Sens., 11, 2417-2428, https://doi.org/ 10.1109/JSTARS.2018.2836661.

$-,-,-\longrightarrow,-$, and G. Zhang, 2021: CMODH validation for C-band synthetic aperture radar $\mathrm{HH}$ polarization wind retrieval over the ocean. IEEE Geosci. Remote Sens. Lett., 18, 102-106, https://doi.org/10.1109/LGRS.2020.2967811.

Mears, C. A., D. K. Smith, and F. J. Wentz, 2001: Comparison of Special Sensor Microwave Imager and buoy-measured wind speed from 1987 to 1997. J. Geophys. Res., 106, 11 719-11 729, https://doi.org/10.1029/1999JC000097.

Mouche, A., D. Hauser, J.-F. Daloze, and C. Guerin, 2005: Dualpolarization measurements at C-band over the ocean: Results from airborne radar observations and comparison with Envisat ASAR data. IEEE Trans. Geosci. Remote Sens., 43, 753-769, https://doi.org/10.1109/TGRS.2005.843951.

—, B. Chapron, B. Zhang, and R. Husson, 2017: Combined coand cross-polarized SAR measurements under extreme wind conditions. IEEE Trans. Geosci. Remote Sens., 55, 6746-6755, https://doi.org/10.1109/TGRS.2017.2732508.

Nghiem, S. V., S. H. Yueh, R. Kwok, and F. K. Li, 1992: Symmetry properties in polarimetric remote sensing. Radio Sci., 27, 693711, https://doi.org/10.1029/92RS01230.

Nord, M. E., T. L. Ainsworth, J.-S. Lee, and N. J. S. Stacy, 2009: Comparison of compact polarimetric synthetic aperture radar modes. IEEE Trans. Geosci. Remote Sens., 47, 174-188, https://doi.org/10.1109/TGRS.2008.2000925.

Peixoto, J. P., and A. H. Oort, 1992: Physics of Climate. American Institute of Physics, $520 \mathrm{pp}$.

Raney, R. K., 2007: Hybrid-polarity SAR architecture. IEEE Trans. Geosci. Remote Sens., 45, 3397-3404, https://doi.org/ 10.1109/TGRS.2007.895883.

Stoffelen, A., and D. Anderson, 1997: Scatterometer data interpretation: Estimation and validation of the transfer function CMOD4. J. Geophys. Res., 102, 5767-5780, https://doi.org/ $10.1029 / 96 \mathrm{JC} 02860$.

Thompson, A. A., 2015: Overview of the RADARSAT Constellation Mission. Can. J. Remote Sens., 41, 401-407, https://doi.org/ 10.1080/07038992.2015.1104633.

Thompson, D. R., T. M. Elfouhaily, and B. Chapron, 1998: Polarization ratio for microwave backscattering from ocean surface at low to moderate incidence angles. 1998 IEEE Int. Geosci. Remote Sens. Symp., Los Alamitos, CA, Institute of Electrical and Electronics Engineers, 1671-1673, https://doi.org/ 10.1109/IGARSS.1998.692411.

Vachon, P. W., and F. W. Dobson, 2000: Wind retrieval from RADARSAT SAR images: Selection of a suitable C-band HH polarization wind retrieval model. Can. J. Remote Sens., 26, 306-313, https://doi.org/10.1080/07038992.2000.10874781.

_ , and J. Wolfe, 2011: C-band cross-polarization wind speed retrieval. IEEE Geosci. Remote Sens. Lett., 8, 456-459, https:// doi.org/10.1109/LGRS.2010.2085417.

Young, I. R., and A. Ribal, 2019: Multiplatform evaluation of global trends in wind speed and wave height. Science, 364, eaav9527, https://doi.org/10.1126/science.aav9527.

Zhang, B., and W. Perrie, 2012: Cross-polarized synthetic aperture radar: A new potential measurement technique for hurricanes. Bull. Amer. Meteor. Soc., 93, 531-541, https://doi.org/10.1175/ BAMS-D-11-00001.1.

—_ _ _ and Y. He, 2011: Wind speed retrieval from RADARSAT-2 quad-polarization images using a new polarization ratio model. J. Geophys. Res., 116, C08008, https:// doi.org/10.1029/2010JC006522.

-_ - — , P. W. Vachon, X. Li, W. G. Pichel, J. Guo, and Y. He, 2012: Ocean vector winds retrieval from C-band fully polarimetric SAR measurements. IEEE Trans. Geosci. Remote Sens., 50, 4252-4261, https://doi.org/10.1109/ TGRS.2012.2194157.

$\longrightarrow$, - J. A. Zhang, E. Uhlhorn, and Y. He, 2014: High-resolution hurricane vector winds from C-band dual-polarization SAR observations. J. Atmos. Oceanic Technol., 31, 272-286, https:// doi.org/10.1175/JTECH-D-13-00006.1.

- A. Mouche, Y. Lu, W. Perrie, G. Zhang, and H. Wang, 2019: A geophysical model function for wind speed retrieval from C-band $\mathrm{HH}$-polarized synthetic aperture radar. IEEE Geosci. Remote Sens. Lett., 16, 1521-1525, https://doi.org/10.1109/ LGRS.2019.2905578.

Zhang, G., B. Zhang, W. Perrie, Y. He, H. Li, S. Khurshid, and K. Warner, 2019: C-band right-circular polarization ocean wind retrieval. IEEE Geosci. Remote Sens. Lett., 16, 13981401, https://doi.org/10.1109/LGRS.2019.2898557. 